\title{
Delay reduction of transmission control protocol in WiMAX by prioritizing the acknowledgement packets
}

\author{
Suherman Suherman ${ }^{1, *}$ \\ ${ }^{1}$ Universitas Sumatera Utara, Electrical Engineering Department, Medan, Indonesia
}

\begin{abstract}
Transmission Control Protocol (TCP) provides reliable connection by acknowledging every transmitted packet. The faster the acknowledgement (ACK) packet is received, the quicker the next TCP window is sent. Previous study shows that by prioritizing the ACK packet route, the TCP delay for the whole data transmission is reduced. This article utilized the medium access within WiMAX network, which is the scheduler, to prioritize the ACK packet transmission. The NS-2 evaluations with various data rate transmission show that the WiMAX scheduling to prioritize the ACK packet successfully reduces the whole data transmission delay. In average, a thirty-second video duration takes $755 \mathrm{~ms}$ faster when prioritized ACK is applied.
\end{abstract}

\section{Introduction}

Transmission control protocol (TCP) is the most common transport layer protocol used for end to end connection within internet [1]. TCP provides reliable connection where the calling party initiates connection by using three way handshakes. This is to make sure that the called party is available before data transmission is performed. This also enables both parties to exchange parameters and network capabilities to optimize end to end connection performance. For instance, if the calling party is a mobile device and the called end point is a web server, that the requested page can be optimized for mobile user.

Furthermore, TCP requires acknowledgements (ACK) for all the transmitted packets; this service enables TCP to ensure successful transmission so that protocol is reliable. As nodes and links are varied in speed, number of packets sent before expecting the ACK; so called as window, may vary. This enables TCP to perform flow control [2]. TCP may terminate connection after a transmission session (non-persistent) to avoid opening socket that prone to security attack [3]. This call termination also makes use of three way handshakes [4]. Figure 1 illustrates how TCP works. Every TCP signals are followed by ACK.

Many ways are performed to improve TCP performances. TCP variants are propose in ways of controlling the packet flows [5]. To boost TCP transmission speed, multi connection is proposed [6].

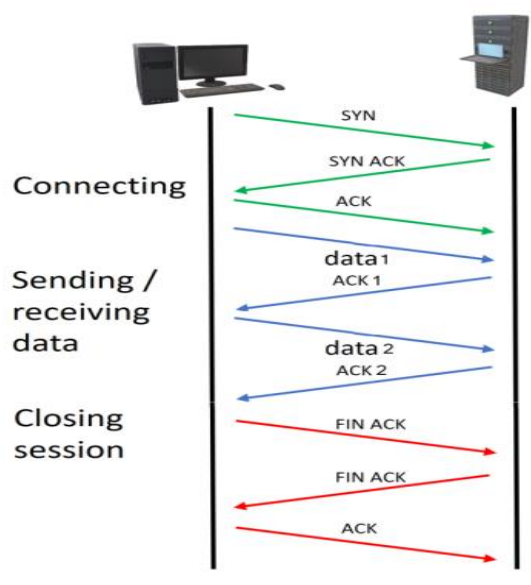

Fig. 1. TCP principle.

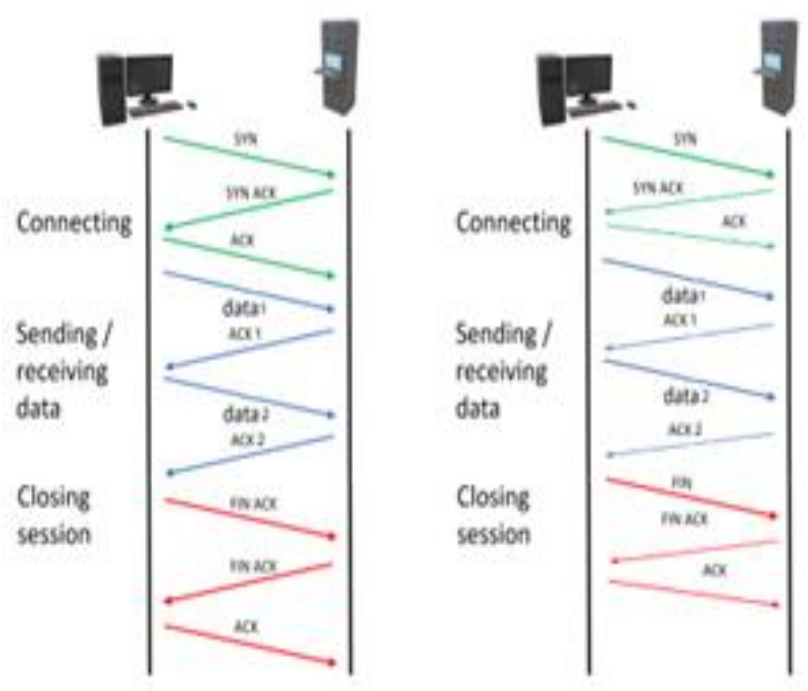

Fig. 2. TCP and the quick ACK.

\footnotetext{
* Corresponding author: suherman@usu.ac.id
} 
Wireless environment induces high packet loss. Wireless TCP is proposed to overcome this matter [7]. In order to avoid multiple retransmissions, TCP-lite was proposed to protect data from unnecessary error [8].

The previous work on reducing the total delay of a bulk data transmission has been proposed by the author [9]. Delay reduction is performed by reducing the ACK transmission. Figure 2 illustrate the ACK delay reduction impact to the overall TCP delay.

In order to allow this to occur, a shorten link designed for ACK transmission was proposed [9]. Instead of experiencing multi-link transmission, ACK is routed to low speed direct link from the called party to the calling party. Figure 2 show the proposed work on previous paper [9]. A direct circuit switching call via cellular is an example of shortened link (Figure 3). It is circuit switching and not the packet switching such as EDGE or HSPA, so that end to end connection for ACK does not experience queue.
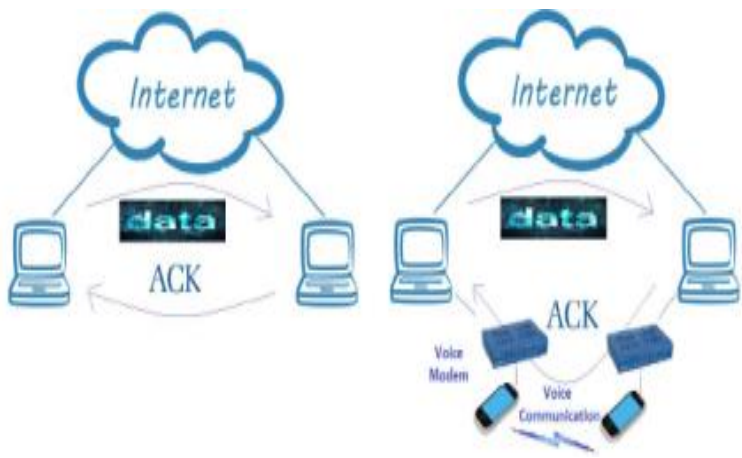

Fig. 3. Sorted ACK route [9].

The results show that direct link exerts TCP delay reduction. Route shortening producing multi-link delay reduction. ACK fastening may be performed in a single link such as in 802.11. Previous research applied ACK delay reduction on 802.11 by reducing the inter-frame space for the ACK packet [10]. Figure 4 shows the 802.11 timing that enables ACK reduction.

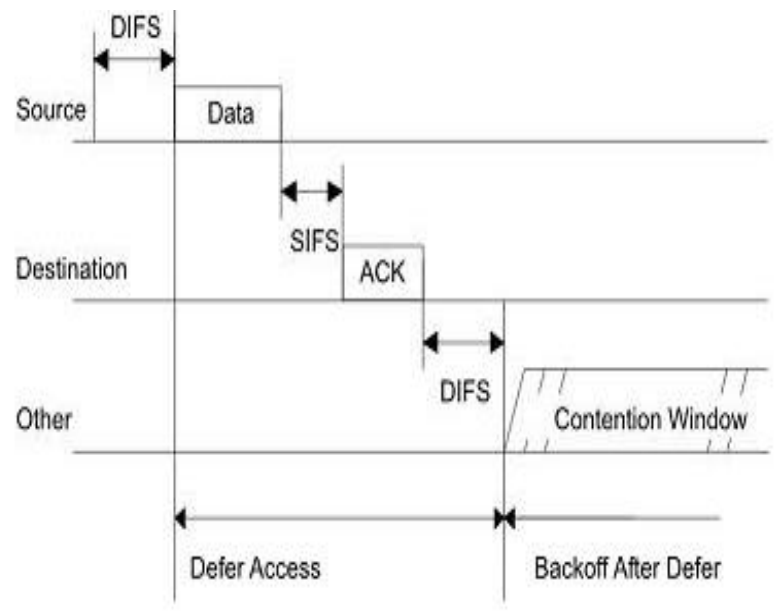

Fig. 4. 802.11 Timing.

This paper proposes TCP delay reduction by using the same technique within an 802.16 network. The 802.16 networks or worldwide interoperability microwave access (WiMAX) provide quality of services (QoS) that differentiates services to clients and traffics [11]. The shortening ACK transmission can be applied by giving the sophisticated service to this packet. This paper however, applies the priority by scheduling scheme that prioritizes the ACK packet. By doing this, it is expected that bandwidth is allocated to ACK before other data type so that ACK delay reduced and TCP transmission fastened.

\section{Research methods}

In order to evaluate the proposed method, NS-2 simulator with WiMAX module from NIST [12] is employed. Although the WiMAX module is limited in features, its BSScheduler is possible for the proposed method to be applied. Due to model limitation, some changes are applied to WiMAX architecture (Figure 5).

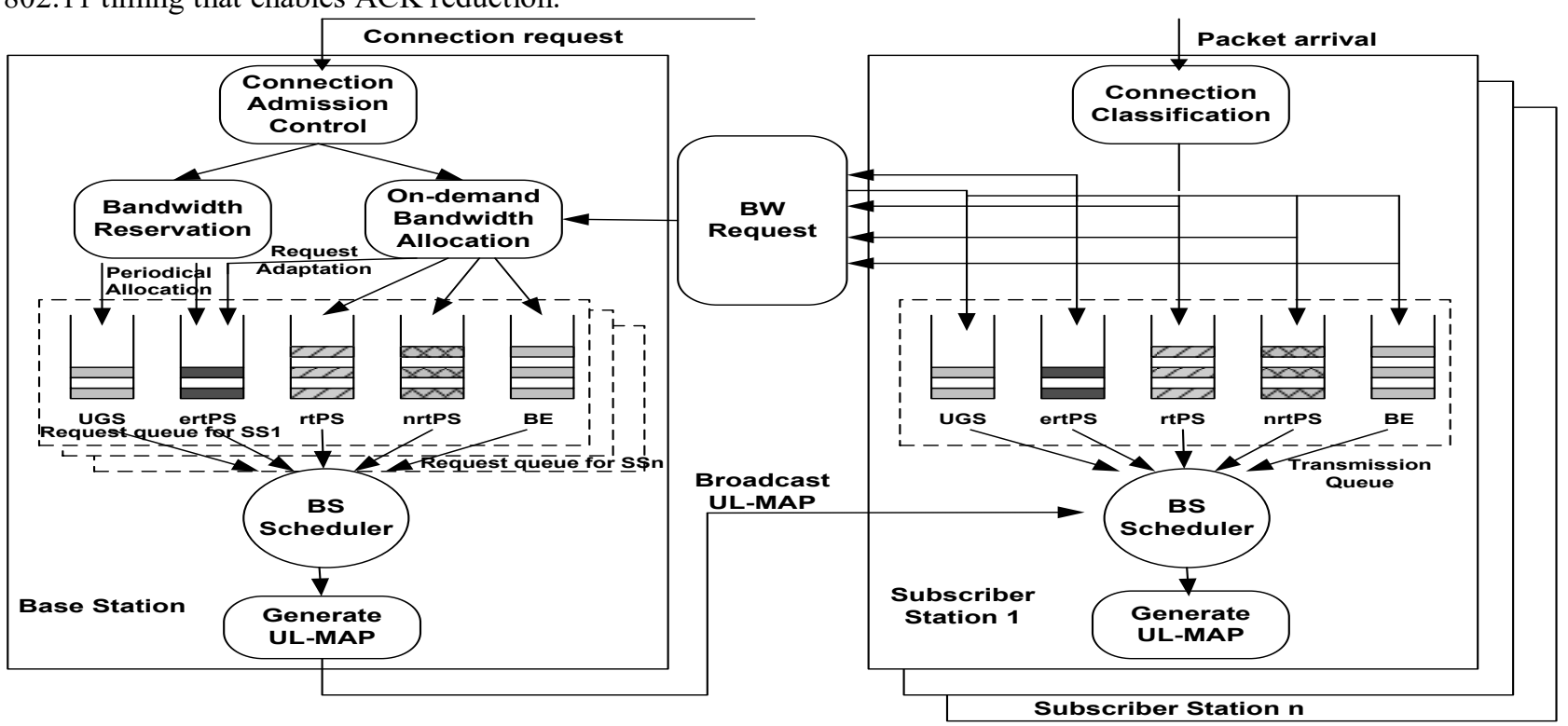

Fig. 5. WiMAX base and subscriber stations architecture [12]. 


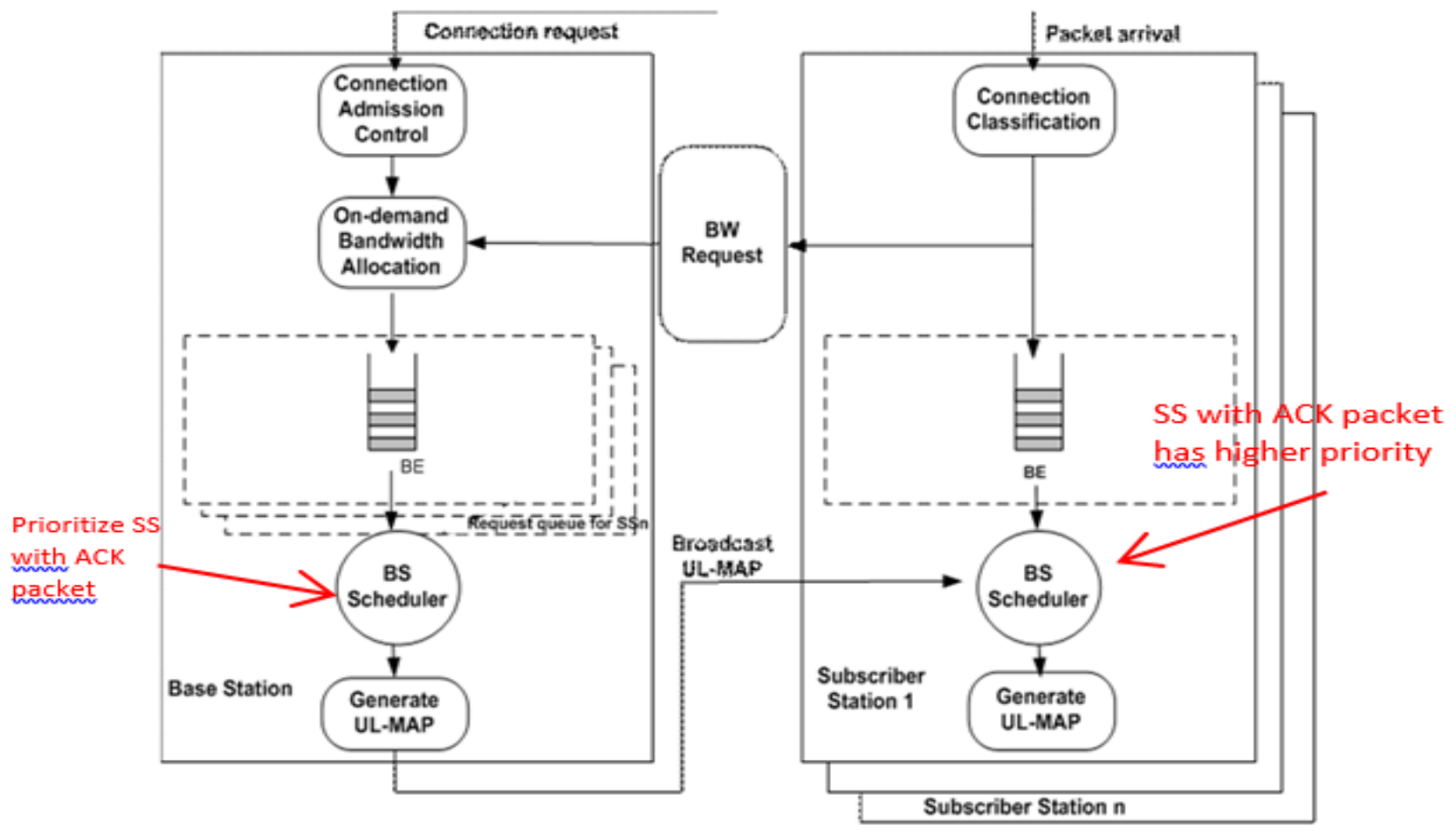

Fig. 6. WiMAX architecture modification.

Normally, WiMAX provides QoS including UGS, ertPS, rtPS, nrtPS, and BE. The only QoS available on NIST module is BE. So the architecture is simplified to single $\mathrm{BE}$ queue and all clients are treated similarly (Figure 6). As there is only $\mathrm{BE}$ available, scheduling algorithm is also simple, applied by using the sorting algorithm based on the type of data, either ACK or data. ACK is prioritized by giving smallest index in it frame header.

Fig. 7 shows the pseudo code for the scheduler that prioritizes ACK packet. Subscriber station (SS) indices are stored in an array. Its properties are accessed by using SS index. Frame type ( $\mathrm{ft} \_$) stores information about ACK. The smallest $\mathrm{ft}$ shows the importance. The $\mathrm{ft}$ of all SSs are sorted from the smallest to the largest.

Bandwidth allocation is based on this sequence, so that the most important packet contains ACK is firstly served.

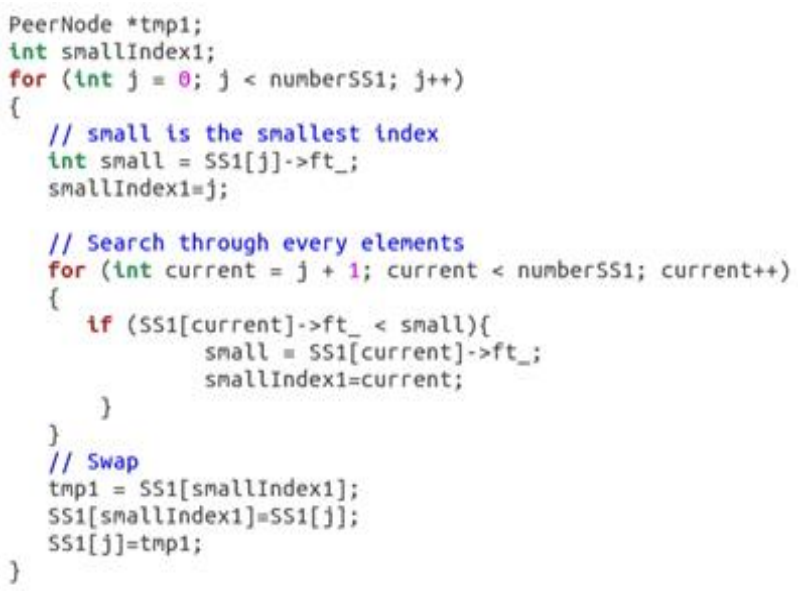

Fig. 7. Scheduler pseudo code.

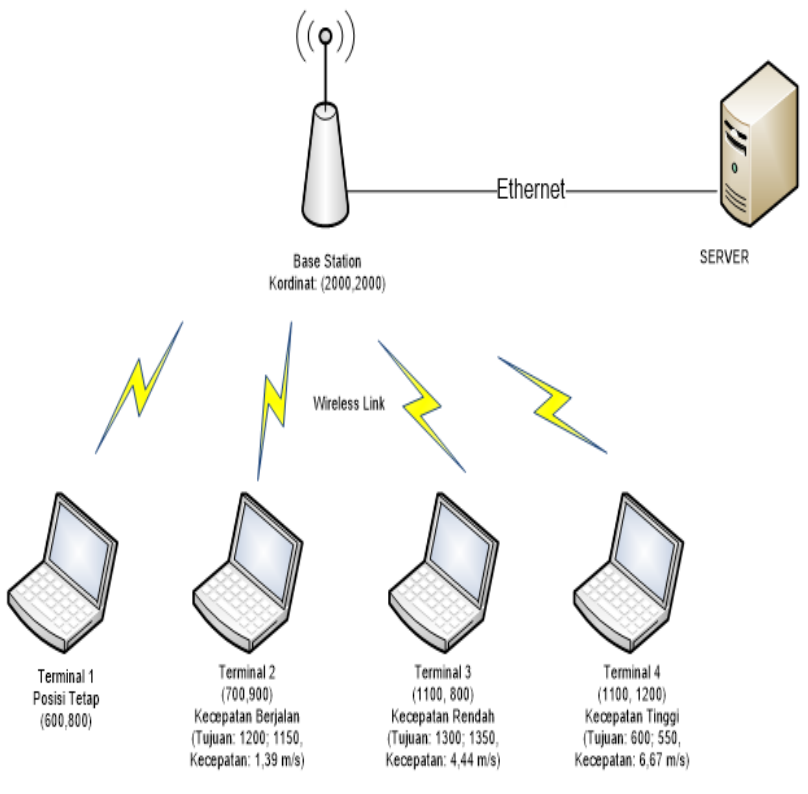

Fig. 8. Network configuration.

Since the NIST module offers only 7 Mbps bandwidth, assessment is limited only to 4 nodes with total bandwidth less than the maximum network throughput. By changing the group of picture (GoP) of the streamed video, traffics have rates about $575 \mathrm{kbps}$ to 1 Mbps. Three nodes are sending traffics uplink, one is downlink. The configuration is shown in Fig. 8.

\section{Evaluation results}

Simulation results show that the proposed method consistently reduce the total TCP transmission delay. 
This is shown in Fig. 9. Delay reduction varies for each traffic assessment. Delay reduction is not affected by the traffic rates.

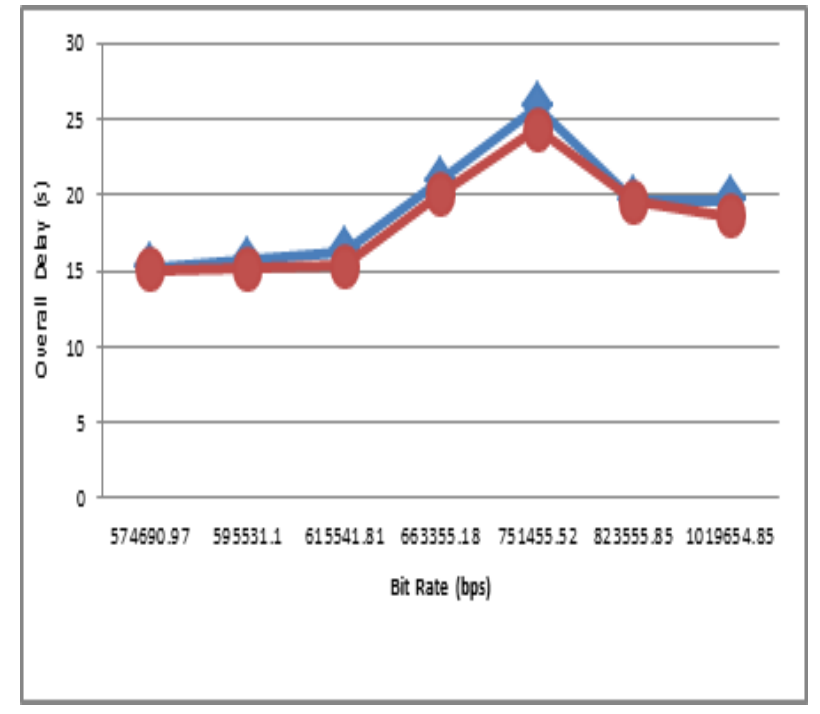

Fig. 9. Overall delay characteristics.

The smallest total delay reduction is $207 \mathrm{~ms}$ at rate $575 \mathrm{kbps}$ and the maximum total delay reduction is 1550 $\mathrm{ms}$ at $750 \mathrm{kbps}$. This result is inline to previous research on 802.11 network [10].

For other parameters, the values are listed in Table 1. Average packet delay is reduced from $167.58 \mathrm{~ms}$ to $166.69 \mathrm{~ms}$. Jitter reduces about $3.16 \mathrm{~ms}$ and packet loss reduces about $0.14 \%$.

Table 1. Other parameters.

\begin{tabular}{|c|c|c|}
\hline Parameters & Before & After \\
\hline Packet Delay & $167.58 \mathrm{~ms}$ & $166.69 \mathrm{~ms}$ \\
\hline Jitter & $35.91 \mathrm{~ms}$ & $32.75 \mathrm{~ms}$ \\
\hline Packet loss & $1.09 \%$ & $0.95 \%$ \\
\hline
\end{tabular}

\section{Conclusions}

By reducing ACK packet transmission time, the next packet is transmitted earlier. As result, total packet transmission from beginning to the end decreases. By prioritizing ACK packet on WiMAX link, data sent by using TCP experiences $0.66 \%$ packet delay reduction; $8.61 \%$ jitter decrement; $0.14 \%$ lower packet losses and $3.82 \%$ quicker transmission.

\section{References}

1. J. Davidson, An introduction to TCP/IP. (Springer Science \& Business Media., 2012)

2. E. G. Lundin, Adaptive relative bitrate manager for
TCP depending flow control, (2017)

3. J. Fielding, R., \& Reschke, Hypertext transfer protocol (HTTP/1.1): Message syntax and routing.

4. T. Kührer, M., Hupperich, T., Rossow, C., Holz, Hell of a Handshake: Abusing TCP for Reflective Amplification DDoS Attacks., WOOT., (2014)

5. V. Abolfazli, E., Shah-Mansouri, Dynamic adjustment of queue levels in TCP Vegas-based networks., Electron. Lett., vol. 52, no. 5, p. 361363., (2016)

6. S. H. Peng, Q., Walid, A., Hwang, J., Low, Multipath TCP: Analysis, design, and implementation., IEEE/ACM Trans. Netw., vol. 24, no. 1, p. 596-609., (2016)

7. S. Dixit, Wireless tcp link state monitoring based video content adaptation and data delivery, U.S. Pat. Appl., vol. No. 15/186, (2016)

8. N. Abdurrahman, H., Sitompul, O. S., Mubarakah, UDP-Lite Enhancement Through Checksum Protection., IOP Conf. Ser. Mater. Sci. Eng., vol. 180 no. 1, p. 12146, (2017)

9. E. Sjani, M., Andriani, Reducing multimedia transmission delay by shortening TCP acknowledgement route, Technol. Inf. Commun. (ISemantic), Int. Semin. Appl. (pp. 114-117). IEEE., (2016)

10. Suherman, Reducing Transmission Control Protocol Delay in 802.11 Network by Prioritizing the Acknowledgement Packets, in The 6th International Conference on Information Technology for Cyber and IT Service Management (CITSM), (2018)

11. M. Suherman, S., Al-Akaidi, Increasing uplink broadband video streaming protocol performance in WiMAX network., Int. J. Internet Protoc. Technol., vol. 7, no. 3, pp. 176-185, (2013)

12. S. Marwan Al-Akaidi, A transport layer protocol for uplink WiMAX video streaming, Int. J. Multimed. Ubiquittous Eng., vol. 10, no. 1, pp. 1932, (2015) 